\title{
Love-Type Wave in PMN-PT Single Crystal Layered Structures with Periodic Undulations
}

\author{
Guoquan Nie \\ School of Mechanical Engineering, Shijiazhuang Tiedao University, Shijiazhuang, 050043, China. \\ Kaike Zhang and Jinxi Liu \\ Department of Engineering Mechanics, Shijiazhuang Tiedao University, Shijiazhuang, 050043, China.
}

(Received 5 August 2018; accepted 19 March 2019)

\begin{abstract}
Love-type wave propagation has been studied in a layered structure consisting of a thin layer of PMN-PT single crystal deposited on an elastic half-space. The weakly periodic undulations of the upper surface of the layered system and the common interface between the PMN-PT layer and the elastic substrate are considered. The PMNPT single crystal is poled along the $[011]_{\mathrm{c}}$ direction so that the macroscopic symmetry is orthonormal $\mathrm{mm} 2$. The dispersion relations for electrically open and shorted circuits are obtained. The effects of various parameters including corrugation, undulatory, position and electrical boundary conditions on dispersion properties of Lovetype waves are discussed. The results show that corrugations of the surface and the interface play a dominant role in the propagation of a Love-type wave, especially, the interfacial corrugation can produce an anomalous dispersion in the case of an electrically shorted condition. The surface corrugation enhances the phase velocity of a Love-type wave while the interface corrugation reduces the phase velocity in the range of relatively smaller wavenumber, and these variations gradually tend to vanish with increase in wavenumber. The undulatory parameter and the position parameter present a secondary impact on the dispersion behaviors of a Love-type wave compared with the corrugation parameters, both of them slightly enhance the phase velocity. The obtained results can offer some fundamentals for improving the efficiency and sensitivity of the interface response in the design and application of piezoelectric SAW devices.
\end{abstract}

\section{INTRODUCTION}

Since the extremely high electric field induced strains and superior electromechanical coupling properties were discovered in relaxor-based ferroelectric lead magnesium niobatelead titanate single crystals (PMN-PT) by Park and Shrout in $1997,{ }^{1,2}$ this series of single crystals have held great promise as candidates for the applications in the next generation of high performance electromechanical devices, including broadband ultrasonic transducers, miniaturized ultrasonic motors, large strain actuators, higher sensitivity sensors, wideband filter devices as well as in the fields of medical ultrasonic for diagnostics and therapy, nondestructive evaluations, structural health monitoring, signal processing and underwater acoustic system, to name a few. ${ }^{3-6} \mathrm{Up}$ to date, the longitudinal piezoelectric strain coefficient $d_{33}(>2800 \mathrm{pC} / \mathrm{N})$ and the electromechanical coupling factor $k_{33}(\sim 94 \%)$ have been confirmed in PMN-0.33PT single crystals at room temperature when it is poled along the $[001]_{\mathrm{c}}$ cubic direction, where the subscript $\mathrm{c}$ is the crystallographic orientation referring to the cubic crystal structure. The electric field induced strain has reached the level of $1.7 \%$, and the dielectric loss is less than $1 \% .^{7,8}$ It was also found that the electromechanical coupling factor $k_{32}$ reaches as high as $94 \%$ and the piezoelectric coefficient $d_{32}$ can be $-1883 \mathrm{pC} / \mathrm{N}$ when PMN-0.29PT single crystal is poled along $[011]_{\mathrm{c}}$ direction. ${ }^{9}$ These superior piezoelectric properties of PMN-PT single crystals significantly outperform the state-of-the-art PZT piezoceramics, which may trigger a rev- olutionary improvement in the performance of electromechanical and electroacoustic devices. ${ }^{10}$ Relaxor-PT ferroelectric single crystals have attracted great attention and have been actively studied over the past 20 years. Several review articles have been written on this series of relaxor-PT single crystals and their crystal growth, property characterizations, piezoelectric mechanisms and related applications. , $^{6,10-13}$

Most of the electromechanical and electroacoustic applications for PMN-PT single crystals, such as ultrasonic transducers, ultrasonic motors, resonant sensor, acoustic tweezers, etc., are directly related to the knowledge of various waves propagating in PMN-PT single crystals and correlative configurations. Activity on this topic in recent years has included bulk wave, surface wave and guided wave in PMN-PT single crystals plate, semi-space, layered composite and others. Zhang et al. experimentally determined a complete set of elastic, piezoelectric, and dielectric constants for [001] c poled PMN-0.33PT single crystal by using ultrasonic and resonance methods. ${ }^{7} \mathrm{~A}$ soft shear mode with very slow velocity of $880 \mathrm{~m} / \mathrm{s}$ in [110]c direction and a maximum shear wave velocity of $2882 \mathrm{~m} / \mathrm{s}$ in $[100]_{\mathrm{c}}$ direction were also found. Jiang et al. investigated the high-frequency properties of [001 $]_{\mathrm{c}}$ poled PMN-0.28PT single crystal by using ultrasonic spectroscopy and found that both the attenuation and the velocity dispersion are very small in the frequency range of $50-110 \mathrm{MHz}$ for a longitudinal wave propagating along the $[001]_{\mathrm{c}}$ direction. ${ }^{14}$ This shows that these engineered domain single crystals have great potential for high- 
frequency applications. For guided waves in PMN-PT single crystal plates, Chen et al. studied the orientation dependence and dispersion relations of coupling between Lamb waves and SH waves in PMN-PT single crystal plates. ${ }^{15,16}$ They found that dispersion curves of a $[001]_{c}$ poled PMN-0.33PT plate show multiple crossings between the symmetric and antisymmetric modes of Lamb waves. SH wave modes are not coupled with Lamb wave modes. SH modes have a limiting velocity of $2880 \mathrm{~m} / \mathrm{s}$ at very high frequency when guided waves propagate along the $[100]_{\mathrm{c}}$ direction of a $[001]_{\mathrm{c}}$ poled PMN-0.33PT plate and have a small limiting velocity of $880 \mathrm{~m} / \mathrm{s}$ (corresponding to the soft shear mode of bulk single crystal) when the guided waves are in $[110]_{\mathrm{c}}$ directions. But SH modes are strongly coupled with the Lamb modes in a $[111]_{\mathrm{c}}$ poled PMN-0.33PT plate due to the non-zero elastic stiffness $c_{14} \cdot{ }^{17,18}$ Surface acoustic wave (SAW) devices made of piezoelectric materials are widely used in the fields of sensor technology, signal processing and electronic filters taking advantage of their small size, low cost and broad compatibility. A few articles have been written on the propagation of SAW in PMN-PT single crystals for enhanced performance of SAW devices. Choi et al. experimentally measured the SAW velocity of a PMN-PT single crystal based on the reported material constants. ${ }^{19}$ An approximate agreement between measured and calculated SAW velocities was obtained. Zhang et al. and $\mathrm{Li}$ et al. theoretically studied SAW propagation in $X$-cut, ${ }^{20} Y$-cut and $Z$-cut ${ }^{21}$ PMN-0.33PT single crystals poled along the $[001]_{\mathrm{c}}$ direction. The corresponding phase velocity, electromechanical coupling coefficient and power flow angle of SAW with the propagation angle varying from $0^{\circ}$ to $180^{\circ}$ were analyzed theoretically. They found that PMN-0.33PT single crystals have very low SAW phase velocity and much higher electromechanical coupling factor compared with quartz, LGT and $\mathrm{LiNbO}_{3}$, and the power flow angle can be zero in certain directions. Similar situations were also found in $X$-cut and $Z$-cut of single-domain $[111]_{c}$ poled PMN-0.33PT single crystals. ${ }^{22}$ These excellent properties make a PMN-PT crystal system a promising material for future SAW devices with miniaturized and enhanced performance.

In order to achieve high performance, in many cases, SAW devices often adopt layered structures consisting of a thin layer deposited on a substrate. The more efficient configurations are the forms of a thin piezoelectric film bonded on an elastic substrate or a reverse structure. Huang et al. derived the dispersion relations of Love wave propagation in a $[001]_{\mathrm{c}}$ poled PMN-0.33 PT single crystal substrate carrying a gold electrode film. ${ }^{23}$ They found that there is no cross coupling among Love wave modes and the attenuation of the amplitude of the guided wave is slower than that of PZT-5 piezoceramic. Therefore, PMN-PT single crystal is extremely suitable for making low loss acoustic wave devices with a high operating frequency. Another high sensitivity gravimetric sensor was found in a system consisting of a carbon fiber epoxy composite deposited on a [011] coled PMN-0.29PT single crystal substrate taking advantage of the larger elastic constant $c_{66}^{E}$ and the higher shear wave velocity of PMN-0.29PT. ${ }^{24}$ Wang et al. studied SH waves in a periodic composite structure consisting of PMN$0.28 \mathrm{PT}$ piezoelectric single crystals and polymer by using a global matrix method..$^{25}$ It was shown that the piezoelectric effect has significant influences on the bandgap width of [011] and $[111]_{\mathrm{c}}$ poled PMN-0.28PT composite laminates but has less effect on the band structure of a [001] $]_{\mathrm{c}}$ poled single crystal system. Nie et al. studied SH wave propagation in [001] $]_{\mathrm{c}}$ and $[011]_{\mathrm{c}}$ poled PMN-PT single crystal layered structures under consideration of the influence of an inhomogeneous initial stress and a viscous liquid loading, respectively. ${ }^{26,27}$ The corresponding effects of the stress gradient, viscous coefficient and density of liquid on wave propagation behaviors were fully analyzed.

This work is firstly motivated by the growing interest in PMN-PT single crystal properties and their applications. Better understanding of the behaviors of SAWs propagation in PMN-PT layered structures is very important for the identification and utilization of useful modes from spurious ones in sensing and filter applications. However, available theoretical results for wave propagation in layered system of PMNPT single crystal are still limited and most of them are for plates or half-space. Secondly, consideration of the corrugation boundary surface can provide some simulations for really uneven surfaces since PMN-PT single crystals always have rough contours during their growth and machining processes. In addition, a thin film waveguide with surface corrugations is often used in the development of a traveling wave amplifier and a distributed feedback oscillator. Recently, Chaudhary et al. investigated Rayleigh wave propagation in a piezoelectric layered composite structure with corrugated boundaries. ${ }^{28}$ The initial stress and rotation existing in both a piezoelectric layer and an orthotropic substrate are considered. Singh et al. studied Love-type wave propagation in a corrugated piezoelectric layered structure and a functionally graded piezoelectric layered structure with imperfect bonding. ${ }^{29,30}$ Dispersion relations for both electrically open and shorted boundary conditions were analytically obtained. The above-mentioned works totally focus on polarized ceramics with the transversely isotropic property. In this paper, we study Love-type wave propagation in PMN-PT single crystal layered structures with a corrugated upper surface and a common interface between the PMN-PT layer and the substrate. The $[011]_{\mathrm{c}}$ poled PMNPT single crystal under consideration possesses macroscopic symmetry of orthonormal $m m 2$. To the authors' knowledge, such work has not been done to date.

\section{STATEMENT OF PROBLEM AND BASIC EQUATIONS}

Consider a PMN-PT single crystal layered structure with the profile of periodic undulations as shown in Fig. 1, where a thin layer of PMN-PT single crystal with average thickness of $H$ having weakly undulated boundary surfaces is deposited on an elastic half-space. The propagating direction of a Love-type wave is along the $x$-axis which is placed at the common interface between the PMN-PT layer and the substrate, while the $y$-axis is pointing vertically downward into the half-space substrate. Since the constituent particles of the media are aligned on a line parallel to the $z$-axis, the field quantities and the geometry are independent of the $z$-coordinate $(\partial / \partial z=0)$. We 


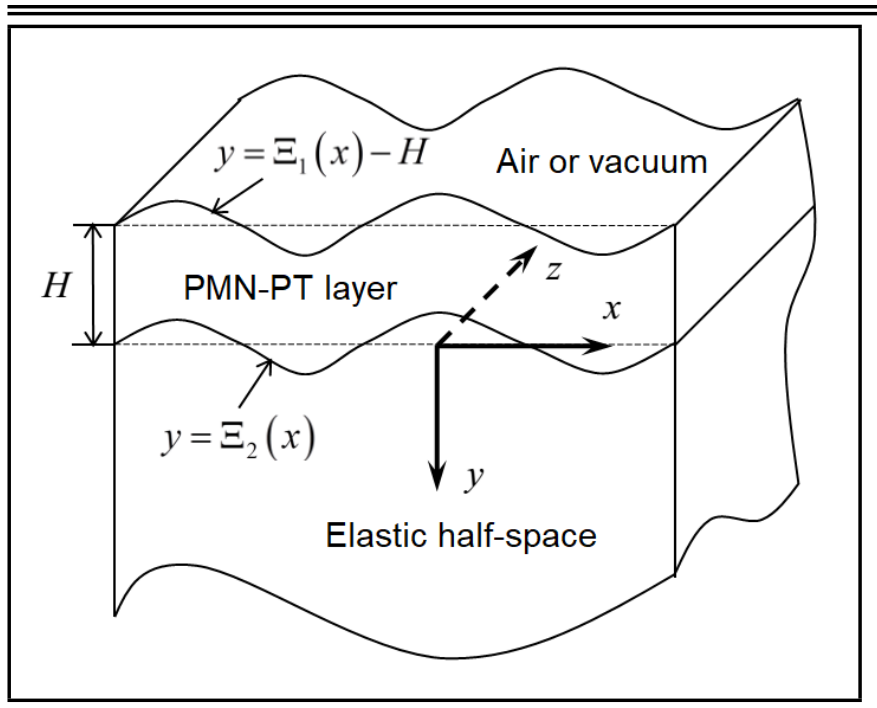

Figure 1. Geometry of a PMN-PT layered structure with undulated boundary surface.

define the upper surface of PMN-PT layer and the common interface between the PMN-PT layer and the substrate in the $y$-direction respectively as $y=\Xi_{1}(x)-H$ and $y=\Xi_{2}(x)$, where $\Xi_{1}(x)$ and $\Xi_{2}(x)$ are periodically continuous functions about $x$-coordinate with

$$
\begin{gathered}
\Xi_{m}(x)=\Xi_{m}(x+\Lambda) \\
\int_{-\Lambda / 2}^{\Lambda / 2} \Xi_{m}(x) d x=0 \\
\Xi_{m}(x)=\Xi_{m}(-x)
\end{gathered}
$$

where $\Lambda$ is the period of the undulated upper surface and the undulated common interface, and $m=1,2$. Since $\Xi_{m}(x)=$ $\sum_{n=1}^{\infty} a_{n}^{m} \cos (n \omega x)$, the shapes of the upper surface and the common interface are both considered to be the superposition of cosinusoidal corrugations of all the harmonics of the fundamental wave number $\omega=2 \pi / \Lambda$. The various harmonics of the corrugation do not interact and each of cosinusoidal corrugations are treated separately. ${ }^{31}$ Therefore, without losing generality, the upper surface of the PMN-PT layer and the common interface are taken to have a weakly cosinusoidal undulation with respect to the planes $y=-H$ and $y=0$, respectively. By letting $a_{1}^{1}=a$ and $a_{1}^{2}=b$, the upper surface of the PMNPT layer and the common interface are taken to be of the form

$$
y=\Xi_{1}(x)-H=a \cos (\omega x)-H
$$

for the undulated upper surface and

$$
y=\Xi_{2}(x)=b \cos (\omega x)
$$

for the undulated common interface, where $a$ and $b$ are the relative amplitudes or the relative depths of the undulated upper surfaces and the undulated common interface.

For Love-type wave propagation in such a layered structure, we consider the anti-plane deformation where the out-of-plane elastic field is coupled with the in-plane electric field. Let $(u, v, w)$ and $\varphi$ be the components of mechanical displacement and electric potential for the undulated PMN-PT single crystal layer and $\left(u^{\prime}, v^{\prime}, w^{\prime}\right)$ and $\varphi^{\prime}$ be the mechanical displacements and electric potential in the substrate, respectively, the antiplane motions are governed by

$$
\left\{\begin{array}{l}
u=v=0, \quad w=w(x, y, t), \quad \varphi=\varphi(x, y, t) \\
u^{\prime}=v^{\prime}=0, \quad w^{\prime}=w^{\prime}(x, y, t), \quad \varphi^{\prime}=\varphi^{\prime}(x, y, t)
\end{array}\right.
$$

For the relaxor-based ferroelectric PMN-PT single crystal under consideration, the domain structures are sensitive to composition, poling condition, and temperature. Generally speaking, PMN-PT single crystals have the symmetry of rhombohedral $3 m$ at room temperature. The polarization is achieved by applying a polarizing field that is greater than the coercive field along a desired crystallographic direction. Poling along the $[001]_{\mathrm{c}}$ direction induces tetragonal $4 \mathrm{~mm}$ macroscopic symmetry, poling along the $[011]_{\mathrm{c}}$ direction induces orthogonal $m m 2$, and poling along the $[111]_{\mathrm{c}}$ direction has rhombohedral $3 m$ symmetry with the single domain state. ${ }^{8}$ In this paper, we focus on the $[011]_{\mathrm{c}}$ poled PMN-PT single crystals due to their well-known independent large thickness shear piezoelectric coefficients and transverse electromechanical properties. ${ }^{6}$ The constitutive relations for $[011]_{\mathrm{c}}$ poled PMN-PT single crystals are given by

$$
\left\{\begin{array}{l}
\tau_{z y}=c_{44} \frac{\partial w}{\partial y}+e_{24} \frac{\partial \varphi}{\partial y} \\
\tau_{z x}=c_{55} \frac{\partial w}{\partial x}+e_{15} \frac{\partial \varphi}{\partial x} \\
D_{y}=e_{24} \frac{\partial w}{\partial y}-\varepsilon_{22} \frac{\partial \varphi}{\partial y} \\
D_{x}=e_{15} \frac{\partial w}{\partial x}-\varepsilon_{11} \frac{\partial \varphi}{\partial x}
\end{array} ;\right.
$$

where $\tau_{z y}$ and $\tau_{z x}$ are the stresses, $D_{x}$ and $D_{y}$ are the electrical displacements; $c_{44}$ and $c_{55}$ are the elastic constants, $e_{24}$ and $e_{15}$ are the piezoelectric constants, $\varepsilon_{11}$ and $\varepsilon_{22}$ are the dielectric constants of the PMN-PT single crystal, respectively.

With the usual quasi-static approximation, the equations of motion and electrostatics for Love waves in piezoelectric media are given by

$$
\left\{\begin{array}{l}
\frac{\partial \tau_{z x}}{\partial x}+\frac{\partial \tau_{z y}}{\partial y}=\rho \frac{\partial^{2} w}{\partial t^{2}} \\
\frac{\partial D_{x}}{\partial x}+\frac{\partial D_{y}}{\partial y}=0
\end{array} ;\right.
$$

where $\rho$ is the density of the PMN-PT single crystal and $t$ is time.

Substituting Eq. (7) into Eq. (8), we can yield the governing equations for the anti-plane deformations in terms of mechanical displacement and electric potential, as follows:

$$
\left\{\begin{array}{l}
\left(c_{55} \frac{\partial^{2}}{\partial x^{2}}+c_{44} \frac{\partial^{2}}{\partial y^{2}}\right) w+\left(e_{15} \frac{\partial^{2}}{\partial x^{2}}+e_{24} \frac{\partial^{2}}{\partial y^{2}}\right) \varphi=\rho \frac{\partial^{2} w}{\partial t^{2}} \\
\left(e_{15} \frac{\partial^{2}}{\partial x^{2}}+e_{24} \frac{\partial^{2}}{\partial y^{2}}\right) w-\left(\varepsilon_{11} \frac{\partial^{2}}{\partial x^{2}}+\varepsilon_{22} \frac{\partial^{2}}{\partial y^{2}}\right) \varphi=0
\end{array}\right.
$$

In the substrate, the mechanical displacement and the electric potential must satisfy the equation of motion and Laplace's equation according to the general theory of elasticity, i.e.,

$$
\left\{\begin{array}{l}
c_{44}^{\prime} \nabla^{2} w^{\prime}=\rho^{\prime} \frac{\partial^{2} w^{\prime}}{\partial t^{2}} \\
\nabla^{2} \varphi^{\prime}=0
\end{array}\right.
$$

where $c_{44}^{\prime}$ is the shear modulus, $\rho^{\prime}$ is the mass density of the elastic substrate, and $\nabla^{2}=\partial^{2} / \partial x^{2}+\partial^{2} / \partial y^{2}$ is the twodimensional Laplacian operator. 
The wave propagation problem governed by Eqs. (9) and (10) should satisfy the boundary and continuity conditions. The continuity conditions at the corrugated common interface $y=\Xi_{2}(x)$ can be expressed as ${ }^{29}$

$$
\begin{aligned}
w & =w^{\prime} \\
\tau_{z y}-\Xi_{2}^{\prime} \tau_{z x} & =\tau_{z y}^{\prime}-\Xi_{2}^{\prime} \tau_{z x}^{\prime} ; \\
\varphi & =\varphi^{\prime} \\
D_{y} & =D_{y}^{\prime} .
\end{aligned}
$$

At the upper surface of PMN-PT layer, the traction free criterion requires ${ }^{29}$

$$
\tau_{z y}-\Xi_{1}^{\prime} \tau_{z x}=0 \quad \text { at } \quad y=\Xi_{1}-H
$$

where $\Xi_{m}^{\prime}=d \Xi_{m} / d x(m=1,2)$ is the derivative of $\Xi_{m}$ with respect to $x$. With the help of Eqs. (4) and (5), we can get the equivalents of conditions in Eqs. (12) and (15), $\tau_{z y}+b \omega \sin (\omega x) \tau_{z x}=\tau_{z y}^{\prime}+b \omega \sin (\omega x) \tau_{z x}^{\prime}$ and $\tau_{z y}+$ $a \omega \sin (\omega x) \tau_{z x}=0$, respectively.

Since the upper surface of the PMN-PT layer is vacuum or air and its permittivity is much less than that of the piezoelectric solid, the electrical condition at the free surface can be classified into two categories, i.e.,

a. Electrically open circuit: $D_{y}=0$ at $y=\Xi_{1}-H$;

b. Electrically shorted circuit: $\varphi=0$ at $y=\Xi_{1}-H$.

The displacement and electric potential in the substrate tend to zero far from the interface along the positive $y$-direction, i.e.,

$$
w^{\prime} \rightarrow 0, \quad \varphi^{\prime} \rightarrow 0 \quad \text { as } \quad y \rightarrow 0 .
$$

\section{GENERAL SOLUTIONS}

For Love-type waves propagating in piezoelectric media, the solutions to Eq. (9) can be assumed as

$$
\left\{\begin{array}{l}
w(x, y, t)=A e^{-r k y} e^{i k(x-c t)} \\
\varphi(x, y, t)=B e^{-r k y} e^{i k(x-c t)}
\end{array} ;\right.
$$

where $k$ is the wave number, $c$ is the phase velocity, $A$ and $B$ are the unknown constants, and $r$ is the undetermined coefficient.

Substituting Eq. (19) into Eq. (9), one can obtain

$$
\left\{\begin{array}{l}
\left(c_{44} r^{2}-c_{55}+\rho c^{2}\right) A+\left(e_{24} r^{2}-e_{15}\right) B=0 \\
\left(e_{24} r^{2}-e_{15}\right) A+\left(\varepsilon_{11}-\varepsilon_{22} r^{2}\right) B=0
\end{array} .\right.
$$

Equation (20) is a system of linear homogeneous equations with respect to the unknown constants $A$ and $B$. A nontrivial solution requires that the determinant of coefficient matrix must vanish, i.e.,

$$
\left|\begin{array}{cc}
c_{44} r^{2}-c_{55}+\rho c^{2} & e_{24} r^{2}-e_{15} \\
e_{24} r^{2}-e_{15} & \varepsilon_{11}-\varepsilon_{22} r^{2}
\end{array}\right|=0 .
$$

Equation (21) is a four-order equation in undetermined coefficient $r$ with the phase velocity $c$ as an unknown parameter. For a given value of $c$, there are four roots satisfying Eq. (21) denoted by $r_{j}(j=1-4)$. The complete solutions of PMNPT single crystal layer must contain all four roots of $r$, so the displacements and electric potential can be rewritten as

$$
\left\{\begin{array}{l}
w(x, y, t)=\sum_{j=1}^{4} A_{j} e^{-r_{j} k y} e^{i k(x-c t)} \\
\varphi(x, y, t)=\sum_{j=1}^{4} \eta_{j} A_{j} e^{-r_{j} k y} e^{i k(x-c t)}
\end{array} ;\right.
$$

where $\eta_{j}=-\frac{c_{44} r^{2}-c_{55}+\rho c^{2}}{e_{24} r^{2}-e_{15}},(j=1-4)$.

Applying the constitutive relations in Eq. (7), the stress and electric displacement components can be obtained as

$$
\left\{\begin{aligned}
\tau_{z y} & =\sum_{j=1}^{4}\left(-c_{44}-e_{24} \eta_{j}\right) k A_{j} r_{j} e^{-r_{j} k y} e^{i k(x-c t)} \\
\tau_{z x} & =\sum_{j=1}^{4}\left(c_{55}+e_{15} \eta_{j}\right) i k A_{j} e^{-r_{j} k y} e^{i k(x-c t)} \\
D_{y}= & \sum_{j=1}^{4}\left(-e_{24}+\varepsilon_{22} \eta_{j}\right) k A_{j} r_{j} e^{-r_{j} k y} e^{i k(x-c t)} \\
D_{x}= & \sum_{j=1}^{4}\left(e_{15}-\varepsilon_{11} \eta_{j}\right) i k A_{j} e^{-r_{j} k y} e^{i k(x-c t)}
\end{aligned}\right.
$$

The solutions of displacement and electric potential for the substrate can be obtained by taking account of the attenuation condition in Eq. (18), i.e.

$$
\left\{\begin{array}{l}
w^{\prime}(x, y, t)=A_{5} e^{-\lambda^{\prime} k y} e^{i k(x-c t)} \\
\varphi^{\prime}(x, y, t)=A_{6} e^{-k y} e^{i k(x-c t)}
\end{array}\right.
$$

where $A_{5}$ and $A_{6}$ are the unknown constants, $\lambda^{\prime}=$ $\sqrt{1-\left(c / c_{s h}^{\prime}\right)^{2}}$, and $c_{s h}^{\prime}=\sqrt{c_{44}^{\prime} / \rho^{\prime}}$ is the bulk shear wave velocity of the substrate.

The stresses and electric displacements in the substrate are

$$
\left\{\begin{array}{l}
\tau_{z y}^{\prime}=-c_{44}^{\prime} A_{5} \lambda^{\prime} k e^{-\lambda^{\prime} k y} e^{i k(x-c t)} \\
\tau_{z x}^{\prime}=c_{44}^{\prime} A_{5} i k e^{-\lambda^{\prime} k y} e^{i k(x-c t)} \\
D_{y}^{\prime}=\varepsilon_{11} A_{6} k e^{-k y} e^{i k(x-c t)} \\
D_{x}^{\prime}=-\varepsilon_{11} A_{6} i k e^{-k y} e^{i k(x-c t)}
\end{array}\right.
$$

where $\varepsilon_{11}^{\prime}$ is the dielectric constant of the substrate.

\section{DISPERSION RELATIONS}

So far, the solutions for [011 $]_{\mathrm{c}}$ poled PMN-PT single crystal layer and elastic substrate are completely obtained. By taking advantage of the boundary conditions and continuity conditions in Eqs. (11)-(16), a system of six-order linear homogenous equations with unknown constants $A_{n}(n=1-6)$ can be yielded. A nontrivial solution requires that the determinant of the coefficient matrix must vanish, what is given by Eq. (26), where $S_{j}=\left(P_{j}-i Q_{j} \Xi_{1}^{\prime}\right), R_{j}=\left(-e_{24}+\varepsilon_{22} \eta_{j}\right) r_{j}, T_{j}=$ $\left(P_{j}-i Q_{j} \Xi_{2}^{\prime}\right)$, and $P_{j}=\left(-c_{44}-e_{24} \eta_{j}\right) r_{j}, Q_{j}=c_{55}+e_{15} \eta_{j}$, $j=1-4$.

Equation (26) elucidates that the Love-type wave propagates in an undulated PMN-PT single crystal layered structure. The phase velocity of a Love-type wave is not only associated with the material constants but also with the corrugation of surface and interface depicted by the functions $\Xi_{1}(x)$ and $\Xi_{2}(x)$. Also, 


$$
\left|\begin{array}{cccccc}
S_{1} e^{-r_{1} k\left(\Xi_{1}-H\right)} & S_{2} e^{-r_{2} k\left(\Xi_{1}-H\right)} & S_{3} e^{-r_{3} k\left(\Xi_{1}-H\right)} & S_{4} e^{-r_{4} k\left(\Xi_{1}-H\right)} & 0 & 0 \\
R_{1} e^{-r_{1} k\left(\Xi_{1}-H\right)} & R_{2} e^{-r_{2} k\left(\Xi_{1}-H\right)} & R_{3} e^{-r_{3} k\left(\Xi_{1}-H\right)} & R_{4} e^{-r_{4} k\left(\Xi_{1}-H\right)} & 0 & 0 \\
e^{-r_{1} k \Xi_{2}} & e^{-r_{2} k \Xi_{2}} & e^{-r_{3} k \Xi_{2}} & e^{-r_{4} k \Xi_{2}} & -e^{-\lambda^{\prime} k \Xi_{2}} & 0 \\
T_{1} e^{-r_{1} k \Xi_{2}} & T_{2} e^{-r_{2} k \Xi_{2}} & T_{3} e^{-r_{3} k \Xi_{2}} & T_{4} e^{-r_{4} k \Xi_{2}} & c_{44}^{\prime}\left(\lambda^{\prime}+i \Xi_{2}^{\prime}\right) e^{-\lambda^{\prime} k \Xi_{2}} & 0 \\
\eta_{1} e^{-r_{1} k \Xi_{2}} & \eta_{2} e^{-r_{2} k \Xi_{2}} & \eta_{3} e^{-r_{3} k \Xi_{2}} & \eta_{4} e^{-r_{4} k \Xi_{2}} & 0 & -e^{-k \Xi_{2}} \\
R_{1} e^{-r_{1} k \Xi_{2}} & R_{2} e^{-r_{2} k \Xi_{2}} & R_{3} e^{-r_{3} k \Xi_{2}} & R_{4} e^{-r_{4} k \Xi_{2}} & 0 & -\varepsilon_{11}^{\prime} e^{-k \Xi_{2}}
\end{array}\right|=0
$$

$$
\left|\begin{array}{cccccc}
P_{1} e^{r_{1} k H} & P_{2} e^{r_{2} k H} & P_{3} e^{r_{3} k H} & P_{4} e^{r_{4} k H} & 0 & 0 \\
R_{1} e^{r_{1} k H} & R_{2} e^{r_{2} k H} & R_{3} e^{r_{3} k H} & R_{4} e^{r_{4} k H} & 0 & 0 \\
e^{-r_{1} k \Xi_{2}} & e^{-r_{2} k \Xi_{2}} & e^{-r_{3} k \Xi_{2}} & e^{-r_{4} k \Xi_{2}} & -e^{-\lambda^{\prime} k \Xi_{2}} & 0 \\
T_{1} e^{-r_{1} k \Xi_{2}} & T_{2} e^{-r_{2} k \Xi_{2}} & T_{3} e^{-r_{3} k \Xi_{2}} & T_{4} e^{-r_{4} k \Xi_{2}} & c_{44}^{\prime}\left(\lambda^{\prime}+i \Xi_{2}^{\prime}\right) e^{-\lambda^{\prime} k \Xi_{2}} & 0 \\
\eta_{1} e^{-r_{1} k \Xi_{2}} & \eta_{2} e^{-r_{2} k \Xi_{2}} & \eta_{3} e^{-r_{3} k \Xi_{2}} & \eta_{4} e^{-r_{4} k \Xi_{2}} & 0 & -e^{-k \Xi_{2}} \\
R_{1} e^{-r_{1} k \Xi_{2}} & R_{2} e^{-r_{2} k \Xi_{2}} & R_{3} e^{-r_{3} k \Xi_{2}} & R_{4} e^{-r_{4} k \Xi_{2}} & 0 & -\varepsilon_{11}^{\prime} e^{-k \Xi_{2}}
\end{array}\right|=0
$$$$
\left|\begin{array}{cccccc}
S_{1} e^{-r_{1} k\left(\Xi_{1}-H\right)} & S_{2} e^{-r_{2} k\left(\Xi_{1}-H\right)} & S_{3} e^{-r_{3} k\left(\Xi_{1}-H\right)} & S_{4} e^{-r_{4} k\left(\Xi_{1}-H\right)} & 0 & 0 \\
R_{1} e^{-r_{1} k\left(\Xi_{1}-H\right)} & R_{2} e^{-r_{2} k\left(\Xi_{1}-H\right)} & R_{3} e^{-r_{3} k\left(\Xi_{1}-H\right)} & R_{4} e^{-r_{4} k\left(\Xi_{1}-H\right)} & 0 & 0 \\
1 & 1 & 1 & 1 & -1 & 0 \\
P_{1} & P_{2} & P_{3} & P_{4} & c_{44}^{\prime} \lambda^{\prime} & 0 \\
\eta_{1} & \eta_{2} & \eta_{3} & \eta_{4} & 0 & -1 \\
R_{1} & R_{2} & R_{3} & R_{4} & 0 & -\varepsilon_{11}^{\prime}
\end{array}\right|=0
$$

it is observed that Eq. (26) is a set of implicit equations and in complex-valued form due to the energy dissipation induced from the undulated surface and interface of the layered structure. Therefore, the Love-type wave is attenuated, and the wavenumber $k$ should be a complex number. The imaginary part of $k$ represents the attenuation per unit length in the propagation direction. We further separate the real part and imaginary part of Eq. (26), so that the corresponding dispersion relation and attenuation relation for Love-type wave propagation in an undulated PMN-PT layer lying over an elastic half-space in the case of an electrically open circuit can be yielded, respectively. Since the dispersive behaviour of waves is a key issue for the design and operation of piezoelectric acoustic wave devices, we are further interested in the study of the related dispersion characteristics of Love-type wave propagation in such a structure.

With a similar procedure, the dispersion relation for electrically shorted circuit can be obtained by replacing the second equation in Eq. (26) with the following one

$$
\xi_{1} A_{1}+\xi_{2} A_{2}+\xi_{3} A_{3}+\xi_{4} A_{4}=0
$$

where $\xi_{j}=\eta_{j} e^{-r_{j} k\left(\Xi_{1}-H\right)}$.

Next, we present some discussions by taking the case of electrically open circuit as an example to make deep insights into the derived dispersion relations.

Particular case 1: When the upper surface of the PMNPT layer is a plane and the interface between PMN-PT layer and elastic substrate is continuous and periodic, this special case may be regarded as an analogue type of layered structure with an imperfect interface. The imperfect bonding can cause a significant degeneration of a piezoelectric acoustic wave device in a practical application. In this way, we have
$\Xi_{1}=0$ and $\Xi_{2}=b \cos (\omega x)$, respectively, and then $\Xi_{1}^{\prime}=0$, $\Xi_{2}^{\prime}=-b \omega \sin (\omega x)$, Eq. (26) reduces to Eq. (28).

Particular case 2: When the upper surface of PMN-PT layer is continuous and periodic, and the common interface is flat, the undulated surface can simulate an uneven surface or rough surface. It is important since no real surface is ideally smooth and a PMN-PT single crystal always has a disordered contour during their growth and machining processes. In this case, we have $\Xi_{1}=a \cos (\omega x)$ and $\Xi_{2}=0$, then Eq. (26) changes into Eq. (29).

Particular case 3: When both the upper surface of PMN-PT layer and the common interface are flat, the layered structure changes into a perfect system without any undulation within the surface and the common interface, i.e., $\Xi_{1}=0$ and $\Xi_{2}=0$. By letting $a=b=0, c_{55}=c_{44}, \varepsilon_{22}=\varepsilon_{11}$ and $e_{24}=e_{15}$ in Eq. (26), an explicit formulation can be obtained after some simplification procedures, i.e.

$$
\tan (\lambda k H)=\frac{c_{44}^{\prime} \lambda^{\prime}\left[\varepsilon_{11}^{\prime}+\left(\varepsilon_{11}+\frac{e_{15}^{2} \varepsilon_{11}^{\prime}}{\varepsilon_{11} c_{44}^{\prime} \lambda^{\prime}}\right) \tanh (k H)\right]}{\bar{c}_{44} \lambda\left[\varepsilon_{11}^{\prime}+\varepsilon_{11} \tanh (k H)\right]} .
$$

Equation (30) gives the dispersion relation of Love wave propagation in a poled piezoelectric ceramic (transversely isotropic) layer perfectly bonded onto a dielectric substrate (isotropic) in case of electrically open circuit. In Eq. (30), $\lambda=$ $\sqrt{\left(c / c_{s h}\right)^{2}-1}$, where $c_{s h}=\sqrt{\bar{c}_{44} / \rho}$ is the bulk shear wave velocity of piezoelectric ceramic, and $\bar{c}_{44}=c_{44}+e_{15}^{2} / \varepsilon_{11}$ is the stiffened elastic constants with the piezoelectric effect.

Particular case 4: $\quad$ By letting $e_{15}=0$ in Eq. (30) we have

$$
\tan (\lambda k H)=\frac{c_{44}^{\prime} \lambda^{\prime}}{c_{44} \lambda}
$$


Equation (31) is the classical dispersion equation of a Love wave. $^{32}$

\section{NUMERICAL RESULTS AND DISCUSSIONS}

In this section, some numerical results are carried out to show the dispersive characteristics of Love-type waves in undulated PMN-PT single crystal layered systems. The single crystal is considered as PMN-0.29PT and poled along the $[011]_{\mathrm{c}}$ direction. The material constants of PMN-0.29PT are: $c_{44}=67 \mathrm{GPa}, c_{55}=14.4 \mathrm{GPa}, e_{15}=17.1 \mathrm{C} / \mathrm{m}^{2}, e_{24}=$ $11.19 \mathrm{C} / \mathrm{m}^{2}, \rho=8090 \mathrm{~kg} / \mathrm{m}^{3}, \varepsilon_{11}=857 \varepsilon_{0}, \varepsilon_{22}=640 \varepsilon_{0}$, and $\varepsilon_{0}=8.854 \times 10^{-12} \mathrm{~F} / \mathrm{m}$ is the dielectric constant of a vacuum. ${ }^{9}$ The elastic substrate is selected as $\mathrm{SiO}_{2}$ and its material constants are: $c_{44}^{\prime}=31.2 \mathrm{GPa}, \varepsilon_{11}^{\prime}=0.33 \times 10^{-10} \mathrm{~F} / \mathrm{m}$, $\rho^{\prime}=2200 \mathrm{~kg} / \mathrm{m}^{3} .{ }^{33}$ To check the effects of the corrugation amplitudes $a$ and $b$, the corrugation wavenumber $\omega$ as well as the position along the propagation direction on phase velocity and group velocity, four dimensionless parameters are introduced through the corrugation parameters $a \omega$ and $b \omega$, the undulatory parameter $\omega H$, and the position parameter $x / H$. In the following discussions, unless otherwise specified, these dimensionless parameters are assigned by $a \omega=0.3, b \omega=0.5$, $\omega H=0.5$, and $x / H=0.02$.

\subsection{Dispersion Property of Love-Type Wave in Undulated PMN-0.29PT Layered Structure}

To deeply understand the dispersion characteristics of a Love-type wave in undulated PMN-PT layered structure, the dispersive curves of phase velocity and group velocity for the first two modes are shown in Fig. 2(a) for an electrically open circuit and Fig. 2(b) for an electrically shorted circuit, respectively, where the horizontal axis is the non-dimensional wave number defined as $K=k H / 2 \pi$, and the vertical axis denotes phase velocity or group velocity of a Love-type wave. The group velocity $c_{g}$ can be calculated through the formula $c_{g}=c+K \cdot d c / d K{ }^{32}$ From Figs. 2(a) and 2(b), it is seen that the phase velocities and the group velocities of the first two modes both start from the velocity of a bulk shear wave in $\mathrm{SiO}_{2}(3765.9 \mathrm{~m} / \mathrm{s})$ regardless of the electrical boundary conditions. The phase velocity and the group velocity of the first mode fall towards the bulk shear wave velocity $(2558 \mathrm{~m} / \mathrm{s})$ of $[001]_{\mathrm{c}}$ poled PMN-0.29PT for an electrically open circuit and the B-G surface wave velocity $(2273.4 \mathrm{~m} / \mathrm{s})$ of PMN-0.29PT for an electrically shorted case, respectively, while the second mode uniformly tends to the bulk shear wave velocity of PMN-0.29PT with the non-dimensional wavenumber increasing. It is noteworthy to mention that, in the case of an electrically shorted circuit, the dispersive curve of the first mode is no longer monotonic, there exists a critical point of the nondimensional wavenumber $K=0.125$ where the dispersion curve of the phase velocity intersects with that of the group velocity $\left(c=c_{g}=1925.4 \mathrm{~m} / \mathrm{s}\right)$. We can conclude that the dispersive curve of the first mode presents a classical (normal) dispersion when $K$ is less than 0.125 due to the fact that the (a)

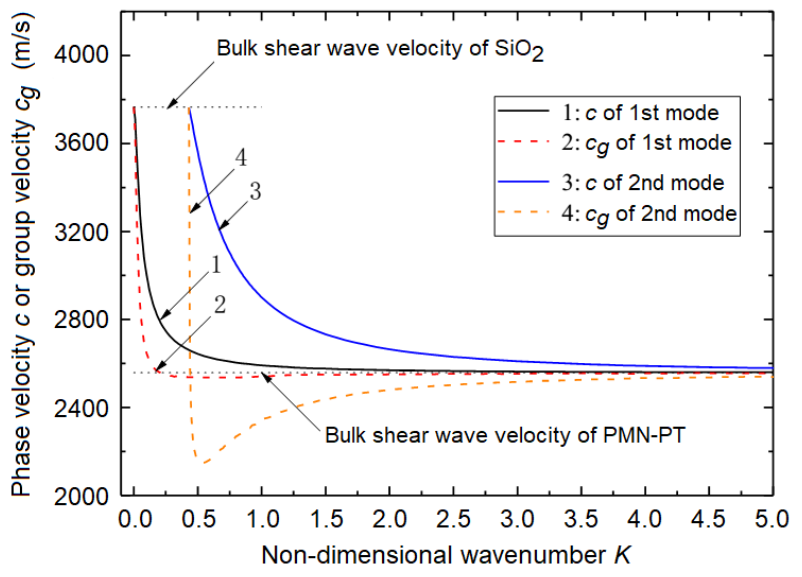

(b)

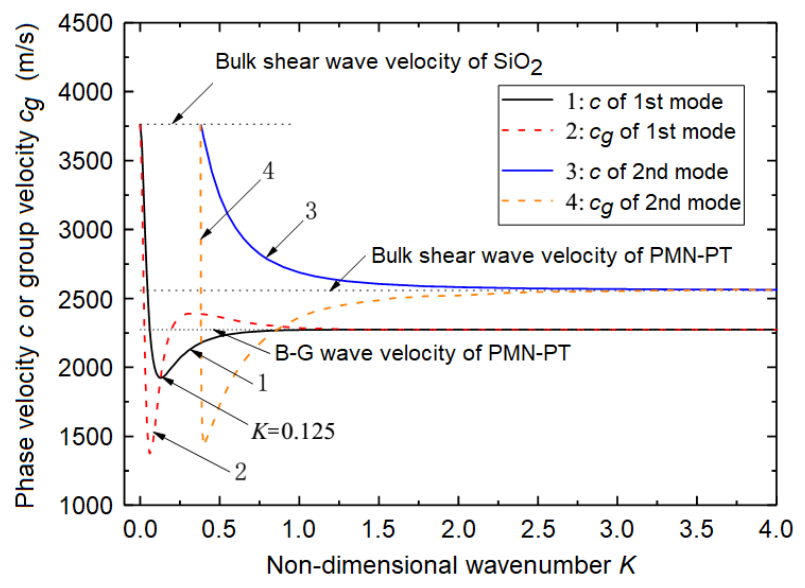

Figure 2. Dispersive curves of phase velocity and group velocity for the first two modes of a Love-type wave in undulated PMN-PT layered structure. (a) electrically open circuit, (b) electrically shorted circuit.

phase velocity is larger than the group velocity for a given $K$. But when $K$ is larger than 0.125 and less than a certain value (about 1.3 for this case, in the range of $K>1.3$, phase velocity always equates to group velocity and there is no dispersion), dispersion is anomalous in this region because the phase velocity is smaller than the group velocity. However, the anomalous dispersion is not found in the electrically open case as well as in the second mode, where the phase velocity is never less than the group velocity.

\subsection{Effect of Corrugation Parameter on Phase Velocity}

Since the lowest mode of a Love-type wave is greatly important in the applications of ultrasonic inspection and measurement and acoustic wave devices, we thus focus on the effects of corrugation parameters on the phase velocity of the first mode. Firstly, we fix the non-dimensional interfacial corrugation parameter $b \omega$ to 0.5 , the values of another non-dimensional surface corrugation parameter $a \omega$ is assigned to $0,0.3,0.5$ and 


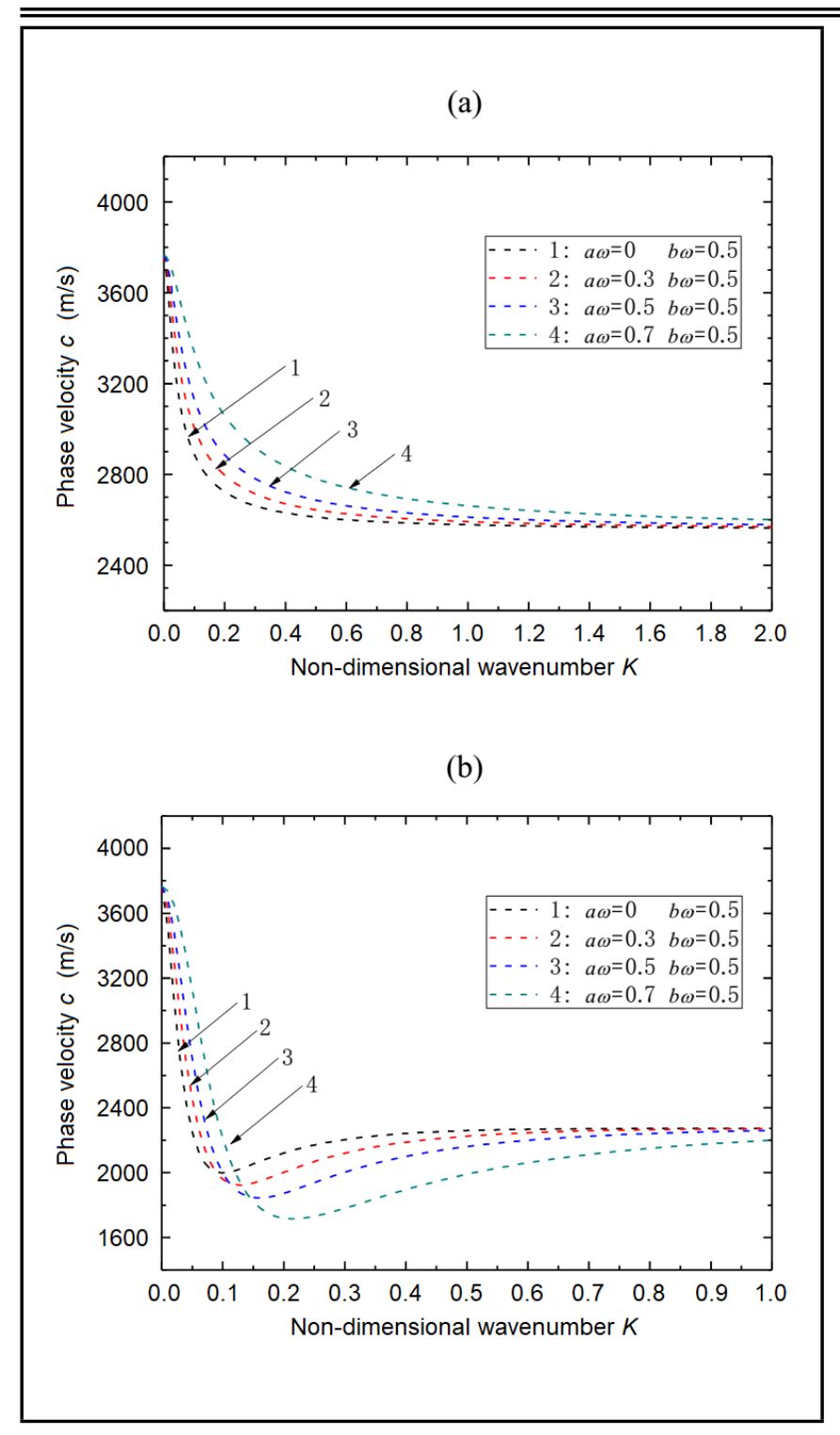

Figure 3. Effect of the corrugation parameter $a \omega$ on the phase velocity of the first mode. (a) electrically open circuit, (b) electrically shorted circuit, where $b \omega=0.5$.

0.7 , respectively, where the case of $a \omega=0$ corresponds to Particular case 1 presented in Section 4. Figures 3(a) and 3(b) depict the effect of the dimensionless parameter $a \omega$ on the phase velocity under electrically open and shorted cases, respectively. It is shown that the phase velocity of the first mode increases with the value of $a \omega$ increasing for a given nondimensional wavenumber $K$ under an electrically open circuit. However, for an electrically shorted circuit, the same variation of phase velocity only occurs at the range of relatively smaller $K$, and the opposite influence shown in the region of larger $K$. It is noticed that the critical values of $K$ corresponding to different values of $a \omega$ which the normal dispersion converts into an anomalous dispersion increase with the value of $a \omega$ increasing, while the corresponding minimum values of phase velocities decrease, the lowest phase velocity drops to $1715.8 \mathrm{~m} / \mathrm{s}$ at $K=0.215$ when $a \omega=0.7$.

Next, we fix the non-dimensional surface parameter $a \omega$ to 0.3 , let $b \omega=0,0.3,0.5$ and 0.7 , respectively, where the case of $b \omega=0$ corresponds to Particular case 2 presented in Section 4. Figures 4(a) and 4(b) illustrate the effect of the di- (a)

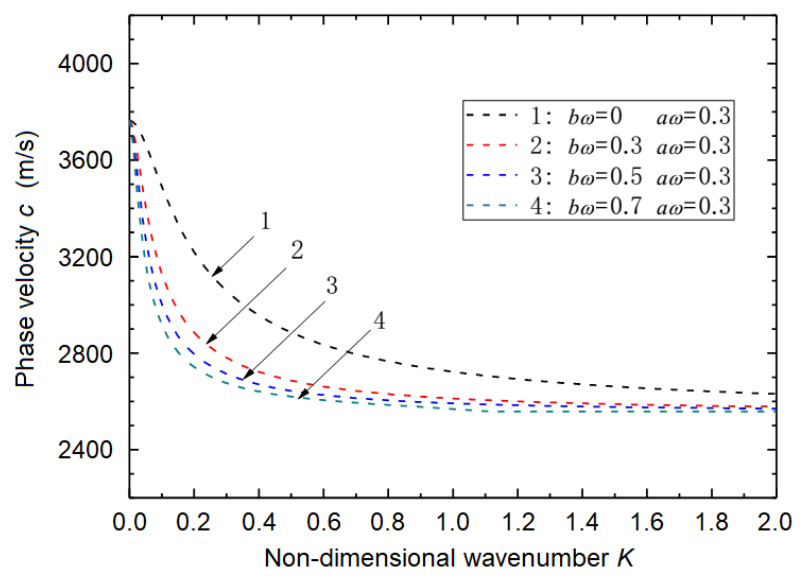

(b)

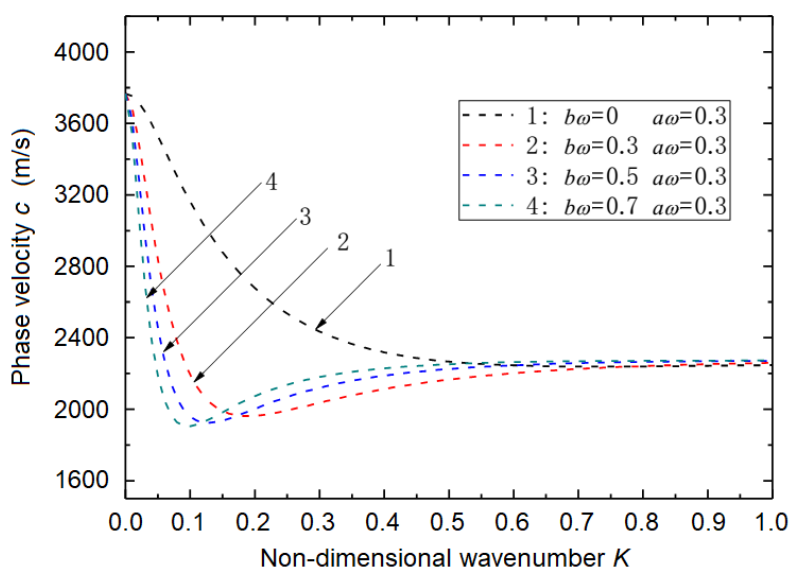

Figure 4. Effect of the corrugation parameter $b \omega$ on the phase velocity of the first mode. (a) electrically open circuit, (b) electrically shorted circuit, where $a \omega=0.3$.

mensionless parameter $b \omega$ on the phase velocity of the first mode under electrically open and shorted cases, respectively. We find an approximately opposite variation of phase velocity induced by $b \omega$ comparing with the impact of aw shown in Fig. 3. This means that the interfacial corrugation can reduce the phase velocity of a Love-type wave while the surface corrugation enhances the phase velocity for electrically open circuit and for electrically shorted circuit in relatively smaller non-dimensional wavenumber. These primary characteristics are completely coincident with those given in Singh, et al. ${ }^{29}$ Moreover, it is found from Curve 1 in Fig. 4(b) that there is no anomalous dispersion when $b \omega=0$ and $a \omega=0.3$. That is to say, the anomalous dispersion existed in case of an electrically shorted circuit is triggered by the corrugation of the common interface between the PMN-PT layer and the substrate (the case when $b \omega \neq 0$ ), while the surface corrugation (the case when $a \omega \neq 0$ ) cannot produce the anomalous dispersion. In view of the significant effects of surface and interface corrugation, we can thus control the phase velocity of a Love-type wave within a required range through the regulation of the cor- 


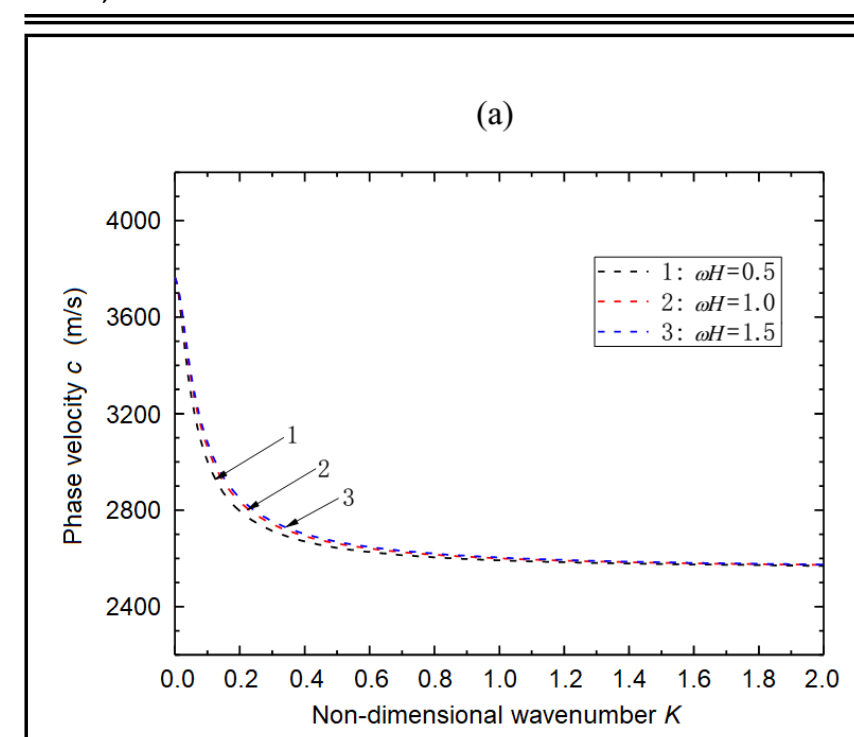

(b)

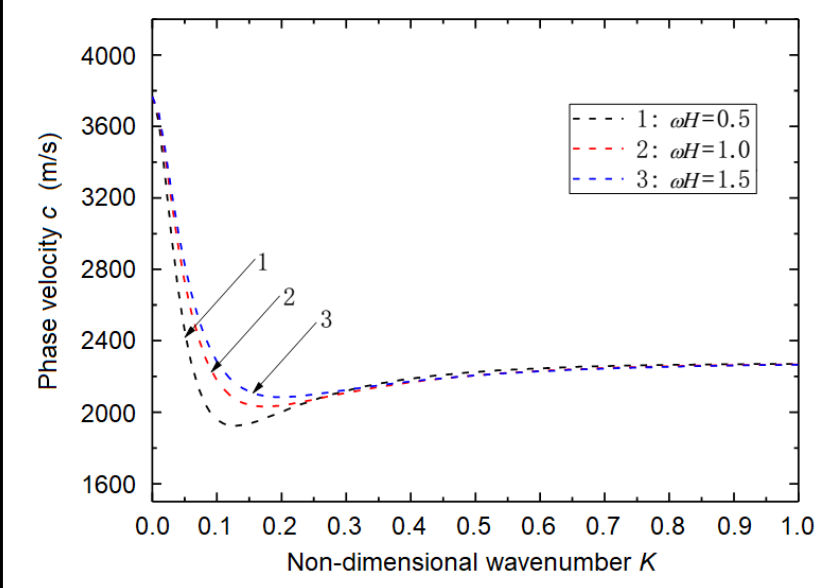

Figure 5. Effect of the undulatory parameter $\omega H$ on the phase velocity of the first mode. (a) electrically open circuit, (b) electrically shorted circuit.

rugation amplitudes to improve the performance of acoustic wave devices, especially for a traveling wave amplifier and a distributed feedback oscillator.

\subsection{Effect of Undulatory Parameter on Phase Velocity}

In order to check the influence of the undulatory parameter $\omega H$ on propagation behavior of a Love-type wave, the dispersion curves of the first mode for different values of $\omega H$ are presented in Fig. 5(a) for an electrically open circuit and Fig. 5(b) for an electrically shorted circuit, respectively, where $\omega H=0.5,1.0$ and 1.5 . It is shown that the undulatory parameter $\omega H$ has larger effect on the phase velocity of an electrically shorted circuit compared with the influence on an electrically open circuit especially in a certain region of a smaller nondimensional wavenumber. A larger value of the undulatory parameter can produce a larger phase velocity for both electrically open and electrically shorted circuits. Compared with the corrugation parameters $a \omega$ and $b \omega$, the undulatory parameter $\omega H$ is a secondary factor in the propagation of a Love-type (a)

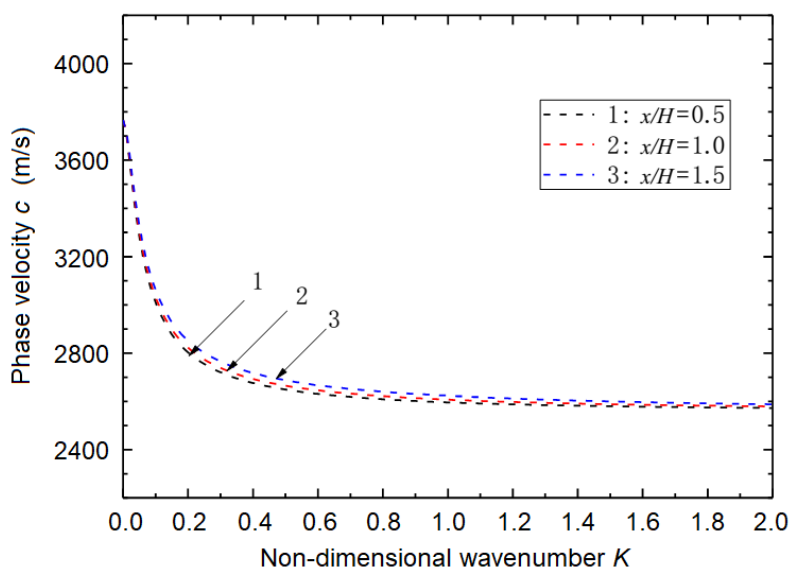

(b)

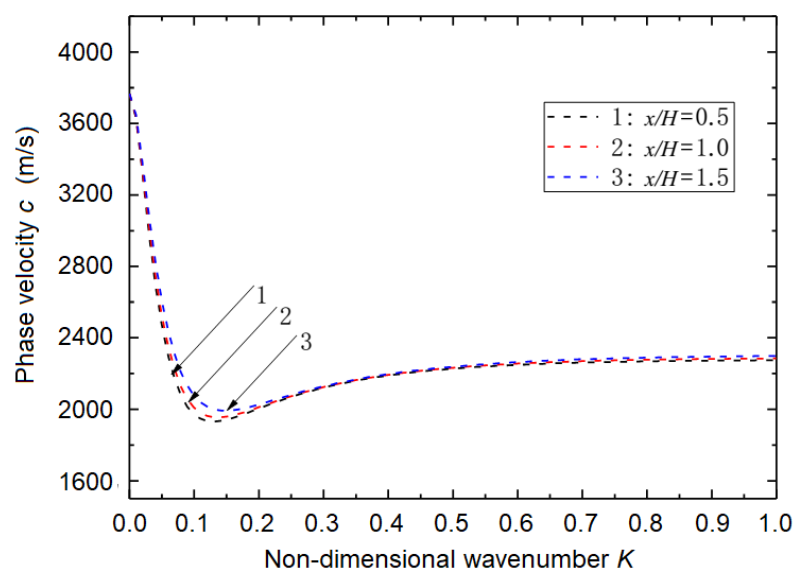

Figure 6. Effect of the position parameter $x / H$ on the phase velocity of the first mode. (a) electrically open circuit, (b) electrically shorted circuit.

wave particularly for the case of an electrically open circuit.

\subsection{Effect of Position Parameter on Phase Velocity}

The effect of the position parameter $x / H$ on the phase velocity of the first mode for electrically open and shorted circuits is presented in Figs. 6(a) and 6(b), respectively, where $x / H=0.5,1.0$ and 1.5 . It is found that the position parameter $x / H$ has less influence on phase velocity compared with those of the corrugation parameters and the undulatory parameter for both electrically open and electrically shorted circuits. In spite of this, we still find that the larger the position parameter is, the higher the phase velocity that can be yielded in the range of relatively smaller non-dimensional wavenumbers. From Figs. 3 to 6, we find that the substantial impacts of the corrugation parameters, the undulatory parameter and the position parameter on the phase velocity are focused on the range of relatively smaller non-dimensional wavenumbers, and the influences tend to disappear with increasing non-dimensional wavenumber. 


\section{CONCLUSIONS}

The propagation characteristic of a Love-type wave in an undulated PMN-PT single crystal layered structure is investigated in this paper. The PMN-PT single crystal under consideration is poled along $[011]_{\mathrm{c}}$ direction so that the macroscopic symmetry is orthonormal $m m 2$. The dispersion relations are derived in closed form by taking account into the corrugations of the surface and the common interface of the layered structure. Four particular cases of the dispersion relation are discussed under consideration of either planar upper surface or planar common interface or both. The obtained dispersion relation completely coincides with the classical Love wave equation when the upper surface and the common interface are both planar in the absence of piezoelectricity of the cover layer. Numerical results show that the corrugation parameters play a dominant role in the propagation of a Love-type wave. The interfacial corrugation can produce an anomalous dispersion for the case of an electrically shorted condition while the surface corrugation cannot. The surface corrugation enhances the phase velocity of a Love-type wave while the interface corrugation reduces the phase velocity for an electrically open circuit and for an electrically shorted circuit in the range of relatively smaller non-dimensional wavenumbers. The undulatory parameter and the position parameter present a secondary impact on the propagation of a Love-type wave compared with the corrugation parameters. The effects of the undulatory parameter and the position parameter on the phase velocity for an electrically shorted circuit are larger than those for an electrically open circuit, both make the phase velocity increase slightly. But the effects of these considered parameters gradually tend to disappear with increasing non-dimensional wavenumber. This study can provide some fundamentals for the design and application of Love wave based devices especially for the characterization of rough contour and disordered boundary surfaces as well as for improving device efficiency and sensitivity.

\section{AKNOWLEDGEMENTS}

This work is supported by the National Natural Science Foundation of China (Nos. 11872041, 11272221 and 11472182). Guoquan Nie also greatly acknowledges the Support Plan for One Hundred Outstanding Innovation Talents in Colleges and Universities of Hebei Province of China (SLRC2017052).

\section{REFERENCES}

1 Park, S. E. and Shrout, T. R. Ultrahigh strain and piezoelectric behavior in relaxor based ferroelectric single crystals, Journal of Applied Physics, 82 (4), 1804-1811, (1997). https://dx.doi.org/10.1063/1.365983

2 Park, S. E. and Shrout, T. R. Relaxor based ferroelectric single crystals for electro-mechanical actuators, Materials Research Innovations, 1 (1), 20-25, (1997). https://dx.doi.org/10.1007/s100190050014
3 Chen, Y., Lam, K. H., Zhou, D., Yue, Q., Yu, Y., Wu, J., Qiu, W., Sun, L., Zhang, C., Luo, H., Chan, H. L. W., and Dai, J. High performance relaxorbased ferroelectric single crystals for ultrasonic transducer applications, Sensors, 14 (8), 13730-13758, (2014). https://dx.doi.org/10.3390/s140813730

4 Jiang, X. N., Kim, J., and Kim, K. Relaxor-PT single crystal piezoelectric sensors, Crystals, 4 (3), 351-376, (2014). https://dx.doi.org/10.3390/cryst4030351

5 Zhou, Q. F., Lam, K. H., Zheng, H. R., Qiu, W. B., and Shung, K. K. Piezoelectric single crystal ultrasonic transducers for biomedical applications, Progress in Materials Science, 66, 87-111, (2014). https://dx.doi.org/10.1016/j.pmatsci.2014.06.001

6 Zhang, S. J., Li, F., Jiang, X. N., Kim, J., Luo, J., and Geng, X. C. Advantages and challenges of relaxor-PbTiO 3 ferroelectric crystals for electroacoustic transducers-A review, Progress in Materials Science, 68, 1-66, (2015). https://dx.doi.org/10.1016/j.pmatsci.2014.10.002

7 Zhang, R., Jiang, B., and Cao, W. W. Elastic, piezoelectric, and dielectric properties of multidomain $0.67 \mathrm{~Pb}\left(\mathrm{Mg}_{1 / 3} \mathrm{Nb}_{2 / 3}\right) \mathrm{O}_{3}-0.33 \mathrm{PbTiO}_{3}$ single crystals, Journal of Applied Physics, 90 (7), 3471-3475, (2001). https://dx.doi.org/10.1063/1.1390494

8 Sun, E. W. and Cao, W. W. Relaxor-based ferroelectric single crystals: Growth, domain engineering, characterization and applications, Progress in Materials Science, 65, 124-210, (2014). https://dx.doi.org/10.1016/j.pmatsci.2014.03.006

9 Wang, F. F., Luo, L. H., Zhou, D., Zhao, X. Y., and Lou, H. S. Complete set of elastic, dielectric, and piezoelectric constants of orthorhombic $0.71 \mathrm{~Pb}\left(\mathrm{Mg}_{1 / 3} \mathrm{Nb}_{2 / 3}\right) \mathrm{O}_{3}-0.29 \mathrm{PbTiO}_{3} \quad$ single crystal, Applied Physics Letters, 90 (21), 212903, (2007). https://dx.doi.org/10.1063/1.2743393

10 Zhang, S. J. and Li, F. High performance ferroelectric relaxor- $\mathrm{PbTiO}_{3}$ single crystals: status and perspective, Journal of Applied Physics, 111 (3), 031301, (2012). https://dx.doi.org/10.1063/1.3679521

11 Luo, N. N., Li, Y. Y., Xia, Z. G., and Li, Q. Progress in lead-based ferroelectric and antiferroelectric single crystals: composition modification, crystal growth and properties, CrystEngComm, 14 (14), 4547-4556, (2012). https://dx.doi.org/10.1039/c2ce06430h

$12 \mathrm{Li}, \mathrm{X}$. B. and Luo, H. S. The growth and properties of relaxor-based ferroelectric single crystals, Journal of the American Ceramic Society, 93 (10), 2915-2928, (2010). https://dx.doi.org/10.1111/j.1551-2916.2010.04107.x

13 Luo, J. and Zhang, S. J. Advances in the growth and characterization of relaxor-PT-based ferroelectric 
single crystals, Crystals, 4 (3), 306-330, (2014). https://dx.doi.org/10.3390/cryst4030306

14 Jiang, W. H., Cao, W. W., and Han, P. D. High-frequency dispersion of ultrasonic velocity and attenuation of singlecrystal $0.72 \mathrm{~Pb}\left(\mathrm{Mg}_{1 / 3} \mathrm{Nb}_{2 / 3}\right) \mathrm{O}_{3}-0.28 \mathrm{PbTiO}_{3}$ with engineered domain structures, Applied Physics Letters, 80 (14), 2466-2468, (2002). https://dx.doi.org/10.1063/1.1468265

${ }^{15}$ Chen, C. W., Zhang, R., and Cao, W. W. Orientation dependence of coupling between Lamb and shear horizontal waves in $(1-x) \mathrm{Pb}\left(\mathrm{Mg}_{1 / 3} \mathrm{Nb}_{2 / 3}\right) \mathrm{O}_{3}-x \mathrm{PbTiO}_{3}$ single crystal plates, 18th IEEE International Symposium on the Applications of Ferroelectric, Xian, China, (2009). https://dx.doi.org/10.1109/isaf.2009.5307609

${ }^{16}$ Chen, C. W. and Xiang, Y. Crossing characteristics of Lamb wave modes in $[001]_{\mathrm{c}}$ and $[011]_{\mathrm{c}}$ polarized $\mathrm{Pb}\left(\mathrm{Mg}_{1 / 3} \mathrm{Nb}_{2 / 3}\right) \mathrm{O}_{3}-\mathrm{PbTiO}_{3}$ crystal plates, Physica B: Condensed Matter, 407 (7), 1099-1103, (2012). https://dx.doi.org/10.1016/j.physb.2012.01.079

${ }^{17}$ Chen, C. W., Zhang, R., Chen, H., and Cao, W. W. Guided wave propagation in $0.67 \mathrm{~Pb}\left(\mathrm{Mg}_{1 / 3} \mathrm{Nb}_{2 / 3}\right) \mathrm{O}_{3}-$ $0.33 \mathrm{PbTiO}_{3}$ single crystal plate poled along $[001]_{\mathrm{c}}$, Applied Physics Letters, 91 (10), 102907, (2007). https://dx.doi.org/10.1063/1.2775093

18 Chen, C. W., Zhang, R., and Cao, W. W. Theoretical study on guided wave propagation in $(1-x) \mathrm{Pb}\left(\mathrm{Mg}_{1 / 3} \mathrm{Nb}_{2 / 3}\right) \mathrm{O}_{3}-$ $x \mathrm{PbTiO}_{3}(x=0.29$ and 0.33$)$ single crystal plates, Journal of Physics D: Applied Physics, 42 (9), 095411, (2009). https://dx.doi.org/10.1088/0022-3727/42/9/095411

${ }^{19}$ Choi, K. H., Jin, H. O., Kim, H. J., and Kim, J. Surface acoustic wave propagation properties of the relaxor ferroelectric PMN-PT single crystal, IEEE Ultrasonics Symposium, Atlanta, USA, (2001). https://dx.doi.org/10.1109/ultsym.2001.991600

${ }^{20}$ Zhang, W., Li, X. M., Zhang, R., Huang, N. X., and Cao, W. W. Numerical calculation of SAW propagation properties at the X-cut of ferroelectric PMN-33\%PT single crystals, Chinese Physics Letters, 26 (6), 064301, (2009). https://dx.doi.org/10.1088/0256-307x/26/6/064301

${ }^{21}$ Li, X. M., Zhang, R., Huang, N. X., Lü, T. Q., and Cao, W. W. Surface acoustic wave propagation in Yand Z-cut $0.67 \mathrm{PbMgNbO}_{3}-0.33 \mathrm{PbTiO}_{3}$ single crystals, Journal of Applied Physics, 106 (5), 054110, (2009). https://dx.doi.org/10.1063/1.3211922

${ }^{22}$ Li, X. M., Zhang, R., Huang, N. X., Lü, T. Q., and Cao, W. W. Surface acoustic wave propagation properties in $0.67 \mathrm{~Pb}\left(\mathrm{Mg}_{1 / 3} \mathrm{Nb}_{2 / 3}\right) \mathrm{O}_{3}-0.33 \mathrm{PbTiO}_{3}$ single crystal poled along [111]c, Applied Physics Letters, 95 (24), 242906, (2009). https://dx.doi.org/10.1063/1.3271775

${ }^{23}$ Huang, N. X., Lü, T. Q., Zhang, R., Wang, Y. L., and Cao, W. W. Guided wave propagation in a gold electrode film on a $\mathrm{Pb}\left(\mathrm{Mg}_{1 / 3} \mathrm{Nb}_{2 / 3}\right) \mathrm{O}_{3}-33 \% \mathrm{PbTiO}_{3}$ ferroelectric single crystal substrate, Chinese Physics Letters, 31 (10), 104302, (2014). https://dx.doi.org/10.1088/0256$307 x / 31 / 10 / 104302$

${ }^{24}$ Huang, N. X., Lü, T. Q., Zhang, R., and Cao, W. W. High sensitivity gravimetric sensor made of carbon fiber epoxy composite on $\mathrm{Pb}\left(\mathrm{Mg}_{1 / 3} \mathrm{Nb}_{2 / 3}\right) \mathrm{O}_{3}-\mathrm{PbTiO}_{3}$ single crystal substrate, Applied Physics Letters, 103 (5), 053507, (2013). https://dx.doi.org/10.1063/1.4817266

25 Wang, Y. L., Zhang, R., Sun, E. W., Song, W., and Cao, W. W. Propagation of shear horizontal waves in laminated composites based on differently orientated poled $0.72 \mathrm{~Pb}\left(\mathrm{Mg}_{1 / 3} \mathrm{Nb}_{2 / 3}\right) \mathrm{O}_{3}-0.28 \mathrm{PbTiO}_{3}$ single crystals, Chinese Physics Letters, 30 (9), 096301, (2013). https://dx.doi.org/10.1088/0256-307x/30/9/096301

${ }^{26} \mathrm{Nie}$, G. Q., Liu, X. L., Liu, J. X., and Fang, X. Q. Effect of an inhomogeneous initial stress on Love wave propagation in $0.67 \mathrm{~Pb}\left(\mathrm{Mg}_{1 / 3} \mathrm{Nb}_{2 / 3}\right) \mathrm{O}_{3}-0.33 \mathrm{PbTiO}_{3}$ single crystal layered structure poled along $[011]_{\mathrm{c}}$, Meccanica, 50 (1), 119132, (2015). https://dx.doi.org/10.1007/s11012-014-0059-y

${ }^{27}$ Nie, G. Q., Liu, J. X., Kong, Y. P., and Fang, X. Q. $\mathrm{SH}$ waves in $(1-x) \mathrm{Pb}\left(\mathrm{Mg}_{1 / 3} \mathrm{Nb}_{2 / 3}\right) \mathrm{O}_{3}-x \mathrm{PbTiO}_{3}$ piezoelectric layered structures loaded with viscous liquid, Acta Mechanica Solida Sinica, 29 (5), 479-489, (2016). https://dx.doi.org/10.1016/s0894-9166(16)30266-x

${ }^{28}$ Chaudhary, S., Sahu, S. A., and Singhal, A. Analytic model for Rayleigh wave propagation in piezoelectric layer overlaid orthotropic substratum, Acta Mechanica, 228 (2), 495529, (2017). https://dx.doi.org/10.1007/s00707-016-1708-0

${ }^{29}$ Singh, A. K., Parween, Z., and Kumar, S. Lovetype wave propagation in a corrugated piezoelectric structure, Journal of Intelligent Material Systems and Structures, 27 (19), 2616-2632, (2016). https://dx.doi.org/10.1177/1045389x 16635843

${ }^{30}$ Singh, A. K., Kumar, S., and Kumari, R. Impact of interfacial imperfection on transverse wave in a functionally graded piezoelectric material structure with corrugated boundaries, The European Physical Journal Plus, 133 (3), 120, (2018). https://dx.doi.org/10.1140/epjp/i2018-11935$\mathrm{x}$

${ }^{31}$ Seshadri, S. R. Coupling of guided modes in thin films with surface corrugation, Journal of Applied Physics, 63 (10), R115-R146, (1988). https://dx.doi.org/10.1063/1.340381

32 Rose, J. L. Ultrasonic Waves in Solid Media, Cambridge University Press, England, (1999).

${ }^{33} \mathrm{Su}$, J., Kuang, Z. B., and Liu, H. Love wave in $\mathrm{ZnO} / \mathrm{SiO}_{2} / \mathrm{Si}$ structure with initial stresses, Journal of Sound and Vibration, 286 (4-5), 981-999, (2005). https://dx.doi.org/10.1016/j.jsv.2004.10.040 